\title{
Simulation of Single Bunch Instabilities Driven by Electron Cloud in the SPS
}

\author{
G. Rumolo, F. Zimmermann, SL/AP, CERN, Geneva, Switzerland
}

\begin{abstract}
Photoemission, or gas ionization, and secondary emission can give rise to a quasi-stationary electron cloud inside the beam pipe through a beam-induced multipacting process. We investigate single bunch instabilities driven by a quasi-stationary electron cloud by means of a computer simulation. The model that we apply makes use of two sets of macroparticles for both the bunch particles and for the electrons, which interact at one or more locations along the beam orbit. Two different schemes have been implemented for the electron cloud field calculation (PIC and soft-Gaussian), and their efficiencies are compared. The code is used to simulate possible instability mechanisms in the SPS. The options of a broad-band wake-field and space charge induced tune spread have been also introduced in order to follow the bunch evolution under the combined effect of the electron-cloud and a broad-band impedance.
\end{abstract}

\section{INTRODUCTION}

A fast vertical instability resulting in beam loss and emittance growth is observed with the LHC proton test beam at the SPS. The instability starts at currents which are just above the threshold of beam-induced multipacting [1], and its progression along a bunch train is reminiscent of the vertical blow up observed at KEKB. The latter has been attributed to a single-bunch instability driven by an electron cloud [2]. The same explanation has been evoked for the SPS instability. It is supported by various observations [3].

\section{SIMULATION PROCEDURES}

To study the instability we have written two simulation programs, which model the interaction of a bunch with an electron cloud over many turns. The beam is represented by $10^{4}$ or $10^{5}$ macroparticles which are distributed in the 6-dimensional phase space. Each macroparticle executes betatron and synchrotron motion. The integrated effect of the electron cloud is localized at one or several interaction points. The electron cloud is also represented by an ensemble of macroparticles. Prior to each bunch passage, a fresh uniform electron cloud is newly generated. The centroid of the cloud is aligned with the beam centroid. The size of the cloud may amount to a few rms beam sizes; boundary conditions from the vacuum chamber have not yet been included. The beam distribution contains deformations which were generated on previous turns. These can be amplified and drive an instability.

To model the interaction of electrons and the beam, the bunch is longitudinally sliced into approximately $20-30$ segments. The interaction of the various slices with the electrons is computed slice by slice. Between slices, the electrons are propagated transversely according to their instantaneous velocity and the length of the slice.

In the first program the forces between bunch and electrons are computed using the standard expression for the transverse field of a Gaussian beam, which involves the complex error function [4]. Each slice of the bunch is approximated by a Gaussian with variable centroid positions and rms sizes. Similar procedures have been used in coherent beam-beam simulations, e.g., in Ref. [5], whence the name 'soft-Gaussian' approximation. Note that for the beam-beam interaction, it has recently been shown that this approximation cannot reproduce the exact mode frequencies [6]. The force of the electrons on the beam is also computed from the Bassetti-Erskine expression by assigning a small Gaussian width, typically one tenth of the beam size, to each macroelectron. The size of the macroelectrons and their number, the number of beam macroparticles, and the size of the cloud can all be varied to study the robustness of the simulation result to numerical fluctuations.

The second program is a particle-in-cell (PIC) code, which has recently been developed. The main difference with the approach described above lies in the way the electric fields from the bunch as well as from the cloud are evaluated. The newly implemented PIC module smears the particles of either species (protons/positrons or electrons) on predefined grid points, and then gets the electric potential at the same points from numerical integration of the Poisson equation. The electric field is therefore computed by simple differentiation and its values are interpolated to the locations where the particles of the other species actually sit. Advancing the particles with this method speeds up the single bunch instability simulations by about a factor 10 with the further advantage of considering a bunch population and an electron cloud of $10^{5}$ macroparticles each (which is typically a factor 5 to 10 larger than the number to which the CPU time had limited us when using the softGaussian approach). Having a higher number of macroparticles also allows for a finer beam slicing. For PIC simulations we normally divide the bunch into 50 slices.

An alternative PIC module was written by K. Ohmi [8].

A third simulation program for the cloud-beam interaction $[2,7]$ represents the bunch by a number of microbunches which are pointlike in longitudinal phase space but feature a constant transverse size equal to the beam size.

Further ingredients which may affect the simulated instability rise times are optionally included, such as a nonzero chromaticity, the conventional transverse broadband impedance of the SPS, which is characterized by 
Table 1: Selected simulation parameters.

\begin{tabular}{lcc}
\hline variable & symbol & value \\
\hline Circumference & $C$ & $6900 \mathrm{~m}$ \\
Beam momentum & $p$ & $26 \mathrm{GeV} / \mathrm{c}$ \\
Chamber half width & $h_{x}$ & $70 \mathrm{~mm}$ \\
Chamber half height & $h_{y}$ & $22.5 \mathrm{~mm}$ \\
Bunch population & $N_{b}$ & $8 \times 10^{10}-10^{11}$ \\
Rms bunch length & $\sigma_{z}$ & $30 \mathrm{~cm}$ \\
Rms energy spread & $\delta p / p_{0}$ & 0.0011 \\
Slip factor & $\eta$ & $5.78 \times 10^{-4}$ \\
Synchrotron tune & $Q_{s}$ & 0.0022 \\
Betatron tune & $Q_{x, y}$ & 26.6 \\
Average beta function & $\beta$ & $15 \mathrm{~m}$ \\
Rms hor. beam size & $\sigma_{x}$ & $3 \mathrm{~mm}$ \\
Rms vert. beam size & $\sigma_{y}$ & $2.3 \mathrm{~mm}$ \\
Electron-cloud density & $\rho_{e}$ & $10^{12} \mathrm{~m}^{-3}$ \\
\hline
\end{tabular}
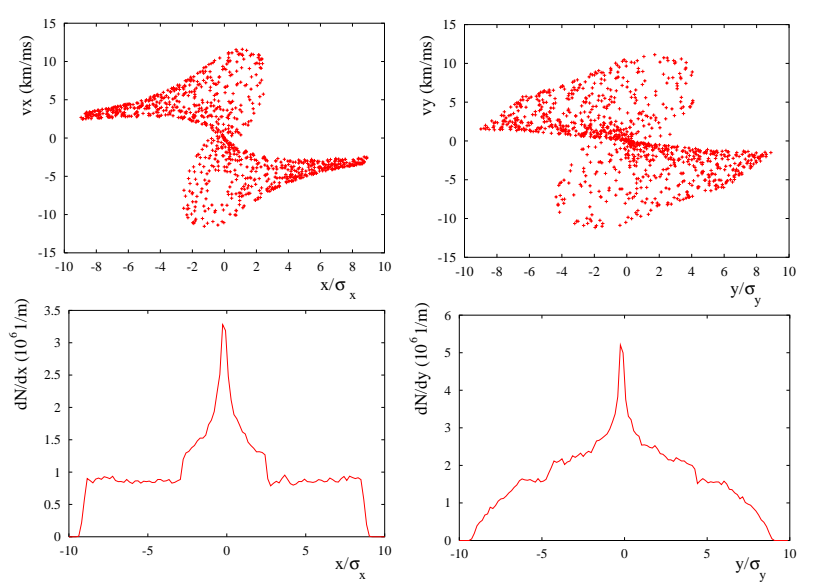

Figure 1: Horizontal and vertical phase space of the electrons after a bunch has gone through (top pictures), and relative distributions (bottom pictures).

$R_{\perp} \approx 30 \mathrm{M} \Omega / \mathrm{m}, f_{r} \approx 1.3 \mathrm{GHz}$ and $Q=1$ [9], and transverse space charge. The maximum incoherent spacecharge tune shift in the SPS at $26 \mathrm{GeV}$ is quite substantial, $\Delta Q_{\mathrm{sc} ; x, y} \equiv c N_{b} e R^{2} /\left(\sqrt{2 \pi} I_{0} \gamma^{3} Q_{x, y} a^{2}\right) \approx-0.015$ (full width), where $I_{0}=31.356 \mathrm{MA}$, and $a \approx 2 \sqrt{\sigma_{x} \sigma_{y}}$ the averaged transverse beam size. In particular, this value is much larger than the synchrotron tune. In fact, the spacecharge tune shift is $z$-dependent, being proportional to the beam line density. The model that we have applied to include its effect in our simulations is the following. At each turn the bunch particles receive a kick at the cloud section, $\Delta x^{\prime}$, (which can optionally include the broad-band equivalent field kick) and then are transported through the ring back to this section by using a matrix that composes a rotation with tune $-\Delta Q_{\mathrm{sc}}(z)$ around the local center of mass $\left(\bar{x}(z), \overline{x^{\prime}}(z)\right)$, the ideal full ring rotation with the nominal tune $Q_{x, y}$, and the chromatic rotation with a momentum dependent tune $\Delta Q_{\operatorname{chr}}(\delta p)$ :

$$
\begin{gathered}
\left(\begin{array}{c}
x_{n+1} \\
x_{n+1}^{\prime}
\end{array}\right)=\mathbf{M}_{\mathrm{chr}}(\delta p) \cdot \mathbf{M}_{\mathrm{ring}} \cdot \\
\cdot\left[\mathbf{M}_{\mathrm{sc}}(z)\left(\begin{array}{c}
x_{n}-\bar{x}(z) \\
x_{n}^{\prime}+\Delta x^{\prime}-\bar{x}^{\prime}(z)
\end{array}\right)+\left(\begin{array}{c}
\bar{x}(z) \\
\overline{x^{\prime}}(z)
\end{array}\right)\right]
\end{gathered}
$$

To avoid unphysical results, the space-charge incoherent tune shift must be included together with the broad-band impedance. The Landau damping that it introduces raises the threshold of the conventional TMCI (otherwise expected for a bunch intensity of $5 \times 10^{10}$ protons), which has never been observed in the SPS operation $[10,11]$.

Table 1 lists relevant simulation parameters.
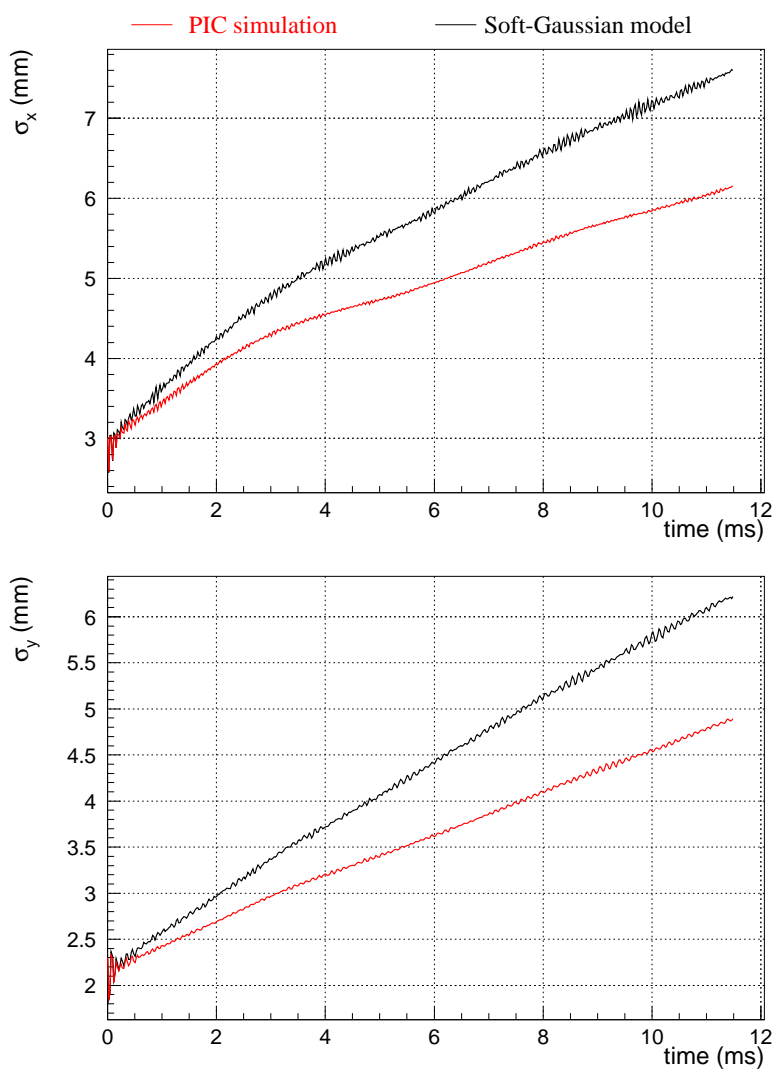

Figure 2: Beam size evolution for an SPS bunch as predicted by PIC and soft-Gaussian simulation $\left(N_{b}=10^{11}\right)$

\section{RESULTS}

As explained above, our code tracks electrons transversely along each bunch passage, and bunch particles over many turns.

The electron motion shows how electrons are progressively focused towards the bunch region as the bunch goes by. In Fig. 1 the electron phase spaces and distributions are shown, as they appear at the end of the interaction with one bunch (having started from transverse uniform distributions). The simulation has been performed here with the PIC code; the agreement with the evolution predicted 
by the soft-Gaussian code is excellent, as may be deduced from the pictures in Ref. [12].

The simulations also yield the evolution of centroid or head-tail motion and the beam emittance growth as a function of time. From Fig. 2 we can deduce that the PIC code foresees growth rates for the beam rms-size smaller than those expected from the soft-Gaussian simulations: over $12 \mathrm{~ms}$ the rms-size increase is about $20 \%$ larger for the latter. This is due to the fact that the incoherent beam enlargement, which occurs in this case where every coherent motion is absent, may be enhanced by the small number of macroparticles used in the soft-Gaussian simulation. Using a higher number of interaction sections might help in this sense but the study is still being carried on. The large emittance growth seems to be consistent with the experimental observations [1].

It is interesting to observe in Fig. 3 how an SPS bunch containing $N_{b}=8 \times 10^{10}$ protons would suffer a strong dipole mode instability under the effect of the broad-band impedance alone, but this effect gets actually damped by space charge and again enhanced by the electron cloud. The instability obviously manifests itself only in the vertical plane, where the impedance has the higher component [9]. A positive chromaticity can strongly damp this effect, as shown in Fig.4.

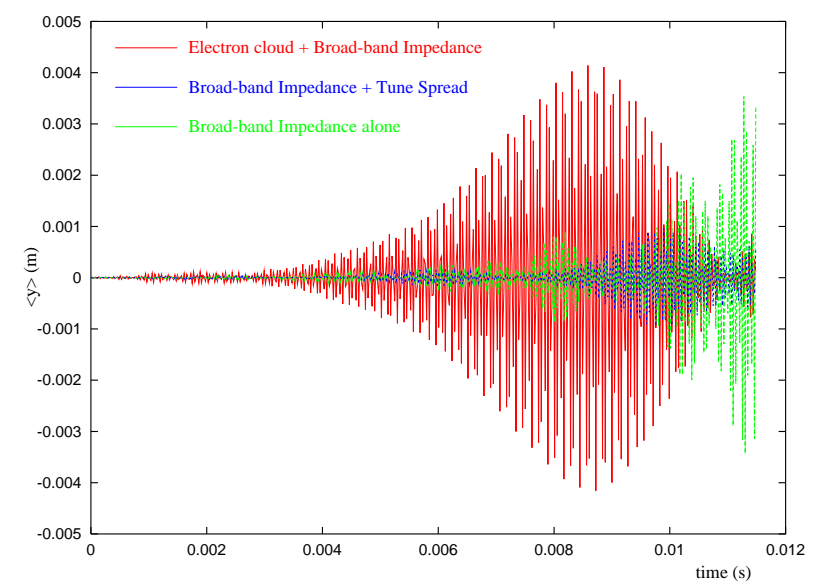

Figure 3: Evolution of the centroid vertical position of an SPS bunch over 500 turns for three different cases.

\section{CONCLUSION}

We have described our methods of simulation of the single bunch instability induced by electron cloud. Having used both a soft-Gaussian approach to the problem and a PIC module, we deduce that a PIC calculation of the electric fields appears convenient in terms of CPU time as well as of accuracy of the results.

We have applied our model to the SPS, including also a broad-band impedance and space charge. A vertical dipole mode instability is found to be triggered by the broad-band impedance alone above a threshold current, and it can be partly stabilized by the space charge driven tune spread,
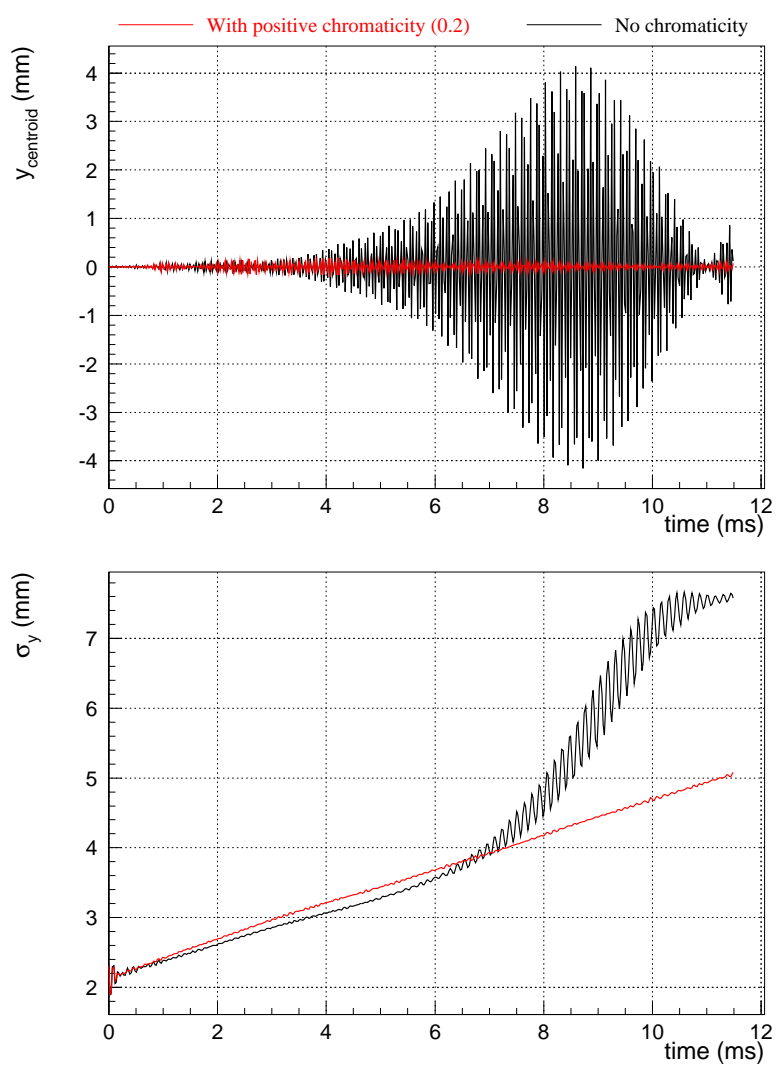

Figure 4: Centroid motion and beam vertical size evolution with and without positive chromaticity (broad-band impedance included).

and also enhanced by the electron cloud. A positive chromaticity can successfully cure this effect.

\section{ACKNOWLEDGEMENTS}

The authors are sincerely indebted to Daniel Schulte and Emiliano Frulloni for the great help and support given in carrying out this work.

\section{REFERENCES}

[1] G. Arduini, et al., "Transverse Behavior of the LHC Proton Beam in the SPS: An Update," these proceedings.

[2] K. Ohmi, F. Zimmermann, Phys. Rev. Lett. 85, 3821 (2000).

[3] G. Arduini, J.M. Jimenez, K. Cornelis, in Proc. Chamonix XI, CERN-SL-2000-007 DI (2001).

[4] M. Bassetti and G. Erskine, CERN/ISR-TH/80-06 (1980).

[5] M.P. Zorzano, F. Zimmermann, PRST-AB 3, 044401 (2000).

[6] K. Yokoya, PRST-AB 3, 124401 (2000).

[7] K. Ohmi, Phys. Rev. E 62, 7287 (2000).

[8] K. Ohmi, G. Rumolo, elsewhere these proceedings.

[9] G. Arduini et al., "Measurements of the SPS Transverse Impedance in 2000", these proceedings.

[10] T. Linnecar, E. Shaposhnikova, SL Note/94-87 (RF) (1994).

[11] M. Blaskiewicz, PRST-AB 1, 044201 (1998).

[12] G. Rumolo, Chamonix XI, CERN-SL-2000-007 DI (2001). 